\title{
Enfermedad neumocócica invasiva en la era posvacunal: Reporte de un caso
}

\author{
Edwin Albino H. Miranda Choque ${ }^{1,2}$ Edgar Jony Lázaro Ignacio ${ }^{3}$
}

\section{RESUMEN}

La enfermedad neumococica invasiva $(E N I)$ es la presentación clínica más severa de la infección por Streptococcus pneumoniae. El Perú desde el año 2008 incluye a la vacuna antineumocócica en el calendario de vacunación nacional. Presentamos un caso de ENI en una niña de 10 años que acude por emergencia con signos meníngeos; además, dolor abdominal, infiltrado hilio basal derecho en la radiografía de tórax, presencia de líquido peritoneal y adenopatía peritoneal en las imágenes de apoyo diagnóstico. Se aísla en los cultivos de LCR y sangre el Streptococcus pneumoniae serotipo $11 \mathrm{~A}$, cepa no vacunable, siendo sensible a ceftriaxona y vancomicina. Recomendamos la necesidad de conocer más sobre la presencia de serotipos de neumococo no vacunal en el contexto clínico-epidemiológico y su rol patogénico en población peruana.

Palabras clave: meningitis, neumococo, vacuna

Invasive pneumococcal disease in the post vaccine era: Case report

\section{ABSTRACT}

Invasive pneumococcal disease (IPD) is the most severe clinical presentation of infection by Streptococcus pneumoniae. Peru since 2008 includes pneumococcal vaccine in the national immunization schedule. We report a case of a 10 year old girl who goes by emergency showing meningeal signs; in addition, abdominal pain, infiltrated right basal hilum on chest radiography, presence of peritoneal fluid and peritoneal adenopathy in diagnostic imaging support. It is isolated in blood and CSF cultures Streptococcus pneumoniae serotype 11A, no vaccine-strain, being sensitive to ceftriaxone and vancomycin. We recommend the need to know more about the presence of not vaccine pneumococcal serotypes in the clinical and epidemiological context and its pathogenic role in Peruvian population.

Keywords: meningitis, pneumococcal vaccine

\footnotetext{
${ }^{1}$ Medico infectólogo del INSN-BREÑA.

${ }_{3}^{2}$ Médico Residente de Pediatría del Hospital Docente Madre Niño San Bartolomé.

${ }^{3}$ Médico Pediatra del servicio de neuropediatría del INSN.
} 


\section{INTRODUCCIÓN}

Streptococcus pneumoniae (también llamada neumococo) es una bacteria Gram positiva, que coloniza la nasofaringe de los humanos, puede ocasionar enfermedad no invasiva como otitis media, sinusitis, neumonía no bacteriémica o puede ocasionar cuadros de enfermedad neumocócica invasiva (ENI) como septicemia, meningitis $(1,2)$. Estas infecciones constituyen un problema importante de salud pública en todo el mundo, siendo la primera causa de muerte por enfermedades inmunoprevenibles; estimaciones de la Organización Mundial de la Salud (OMS) indican que son responsables de causa de muerte de 0,7 a 1 millón de niños menores de 5 años, sobre todo en países de escasos recursos económicos $(3,4)$. En Perú, la bacteria del Neumococo es etiología importante de infecciones de mayor morbilidad general, mayor mortalidad, según el indicador de años de vida potencialmente perdidos, especialmente en los grupos de edad extremos (5).

Se reporta el caso de un paciente con diagnóstico de ENI, con detección de serotipo y susceptibilidad a antibióticos en momentos cuando el Ministerio de Salud usa según el calendario de vacunación la vacuna conjugada antineumocócica (VCP), desde el año $2008(14,15)$.

\section{REPORTE DE CASO}

Paciente femenino de 10 años de edad, natural de lima, estudiante. Ingresa por emergencia del Instituto Nacional de Salud del Niño (INSN), el 30 de junio del 2015, con un tiempo de enfermedad de 3 días caracterizado por cefalea intensa holocraneana, asociado a vómitos alimenticios en 3 veces por día, posterior vómitos biliosos, alza térmica de $38^{\circ} \mathrm{C}$;además, de dolor abdominal. No refiere diarrea o tos. Madre refiere que administró ciprofloxacina 500 mg en 02 oportunidades.

Funciones biológicas: apetito disminuido, deposiciones sin alteración, sueño incrementado, sed incrementada, orina algo disminuido.

Con respectos a los antecedentes, los prenatales y natales sin eventualidades de importancia, con respecto a su alimentación inicial recibió lactancia materna exclusiva. Alimentos complementarios a los 6 meses. Inmunización: vacunas completas para su edad entre el año 2004 al 2005, no refuerzos (no trae cartilla). Desarrollo psicomotor adecuado para su edad; la edad de inicio de escolaridad fue a los 3 años.

Hace 1 año, curso con politraumatismo (traumatismo encefalocraneano con craniectomía descompresiva, trauma de abdomen cerrado, trauma torácico con colocación de tubo de drenaje, trauma renal con hematuria) por lo que fue hospitalizada por 3 meses, siendo trasfundida en 02 oportunidades.

Hace 15 días presentó Infección urinaria hospitalizada en el hospital de Huaycán con tratamiento de ciprofloxacina $500 \mathrm{mg}$ cada 12 horas. No presenta alergias a medicamentos, no antecedentes de contacto con pacientes con tuberculosis, padres y hermanos sanos, resto de antecedente no contributarios.

Al examen físico presentó frecuencia cardiáca de 90 latidos/minuto, frecuencia respiratoria 22 respiraciones/minuto, temperatura de $38.1^{\circ} \mathrm{C}$ (oral), presión arterial $95 / 56 \mathrm{mgHg}$., saturación $\mathrm{O}_{2}$ de $100 \%$, tendencia al sueño. Paciente en mal estado general, regular estado de hidratación, buen estado de nutrición, luce quejumbrosa, ventilando espontáneamente. Piel: tibia elástica hidratada, no ictericia, algo pálida, adenopatías no presenta. Abdomen: blando depresible, dolor en abdomen a la palpación superficial a predominio en fosa ilíaca derecha, impresiona Mac Burney positivo, no se palpa hígado ni bazo debajo del reborde costal, blumberg negativo, RHA presentes. Aparato respiratorio: amplexación conservada, murmullo vesicular para bien en ambos hemitorax. Al examen neurológico: activo reactivo, tendencia al sueño, tono conservado, fuerza muscular conservada, rigidez de nuca, signo de kerning positivo, signo de brudzinsky positivo, signo de babinsky negativo, reflejos osteotendinosos presentes, pupilas isocóricas foto reactivas. El resto del examen sin mayores alteraciones. Se realizó punción lumbar para estudio de líquido cefalorraquídeo (LCR), previa toma de TEM cerebro sin contraste (por antecedentes de TEC severo) y evaluación de fondo de ojo de los cuales fueron informados cómo normales. Se tomaron cultivos de LCR y hemocultivo. Se realizó radiografía de tórax (por antecedentes de traumatismo toráxico) informado como infiltrado alvéolo intersticial en región hilio basal derecho (Figura $1 \mathrm{~A}$ ). 
Figura 1A. Radiografía de tórax, patrón alvéolo intersticial

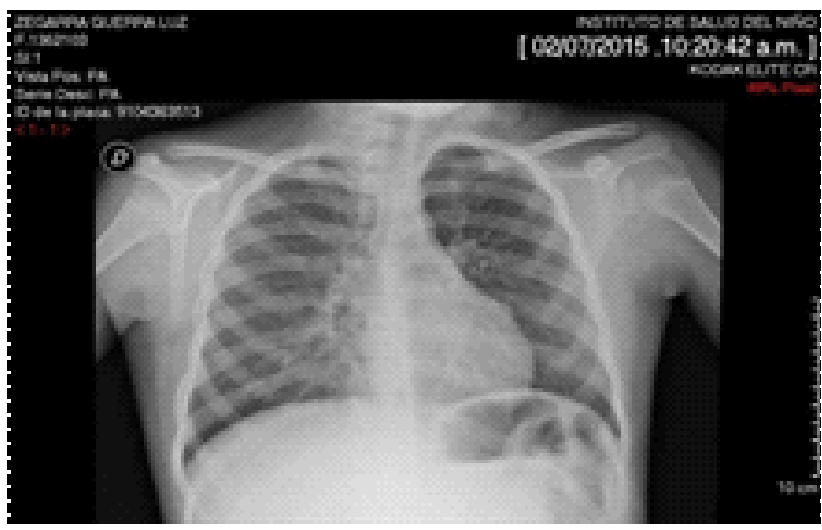

Los exámenes de laboratorio al ingreso hospitalario fueron lo siguiente; en el hemograma: leucocitos de $12,600 \mathrm{cel} / \mathrm{mm}^{3}$, abastonados $9 \%$, segmentados $80 \%$, hemoglobina: $11.6 \mathrm{~g} / \mathrm{dl}$., plaquetas: $256,000 \mathrm{cel} /$ $\mathrm{mm}^{3}$, proteína c reactiva: $26 \mathrm{ng} / \mathrm{dl}$, glicemia 88 $\mathrm{mg} / \mathrm{dl}$, urea $21 \mathrm{mg} / \mathrm{dl}$, creatinina $0.19 \mathrm{mg} / \mathrm{dl}$, ácido úrico $1.8 \mathrm{mg} / \mathrm{dl}$, electrolitos: Na:144 mg/dl, K: 3.4 $\mathrm{mg} / \mathrm{dl}$, cloro: $108 \mathrm{mg} / \mathrm{dl}$., proteínas totales 6.2 $\mathrm{mg} / \mathrm{dl}$, albumina $3.7 \mathrm{mg} / \mathrm{dl}$ (VN 3.2-5.2), globulina $2.5 \mathrm{mg} / \mathrm{dl}$ (VN 2.3-4), transaminasas glutámico pirúvico $134 \mathrm{UI} / \mathrm{L}$, transaminasas glutámico oxalacética $41 \mathrm{UI} / \mathrm{L}$, exámenes de coagulación sin alteración.

Con respecto al examen del líquido cefalorraquídeo (LCR) del 30 de julio 2015: Ligera turbidez con sedimento, leucocitos $116 \mathrm{cel} / \mathrm{mm}^{3}$, monomorfos $56 \%$ polimorfos $44 \%$, proteína $159 \mathrm{mg} / \mathrm{dl}$ glicemia de 0 $\mathrm{mg} / \mathrm{dl}$, test de adenosina de aminasa (ADA): $5.8 \mathrm{U} / \mathrm{L}$. Gram de LCR: diplococos gram positivos lanceolados.

El diagnóstico inicial fue meningitis bacteriana probable germen neumococo. Inició ceftriaxona 2 gramos cada 12 horas vía endovenoso, vancomicina $500 \mathrm{mg}$ cada 6 horas vía endovenoso y dexametasona $0.6 \mathrm{mg} / \mathrm{kg} /$ día vía endovenoso.

Evolucionó favorablemente con ausencia de fiebre y de signos meníngeos a los 3 días, pero con persistencia del dolor abdominal por lo que se solicitó ecografía abdomen el 02 de julio encontrándose líquido libre interasas en fosa ilíaca derecha aproximadamente volumen $1.5 \mathrm{cc}$,e imágenes hipoecoicos mal definidos, el cual el radiólogo sugirió proceso inflamatorio. Fue evaluado por médico de cirugía pediátrica de guardia quienes indicaron conducta expectante y control ecográfico. El dolor abdominal fue menos intenso en los días sucesivos.
El 3 de julio laboratorio del INSN reporta resultados de Hemocultivo y cultivo de LCR, aislándose Streptococcus pneumoniae en las 2 muestras, de los cuales fueron sensibles a cloranfenicol eritromicina, tetraciclinas, vancomicina; y resistente a Cloranfenicol y cotrimoxazol; El serotipo aislado fue el serotipo $11 \mathrm{~A}$ en el laboratorio del Instituto Nacional de Salud de Ministerio de Salud.

Por los hallazgos en la radiografía de tórax y ecografía de abdomen se realizó el 6 de julio una Tomografía Axial Computarizada (TAC) de abdomen y tórax (Figura 1B), donde se evidencia múltiples ganglios en el mesenterio de $12.0 \mathrm{~mm}$ de diámetro, ganglios de $13.4 \mathrm{~mm}$ de diámetro en la región cervical lateral bilateral, no se evidenció liquido libre, resto de órganos con morfología normal.

Figura 1B. TAC abdomen: presencia de adenopatías múltiples mesentéricasen región parahiliar derecho.

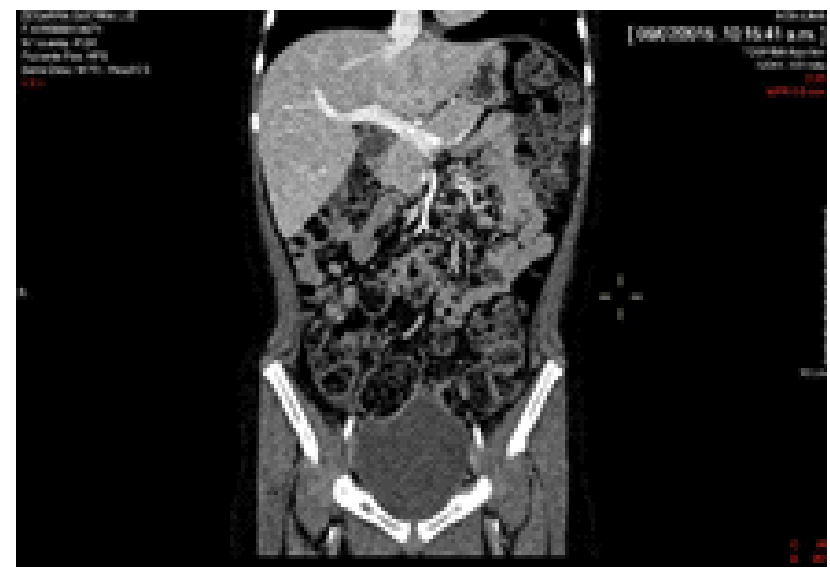

Con los síntomas, los resultados de laboratorio y ayuda diagnóstica, se evidencia daño gastrointestinal, en pulmón y cerebro, siendo el diagnóstico final ENI.

El 11 de julio se realizó control de LCR y hemograma el cual se encontró en el citoquímico un líquido transparente cristal de roca, leucocitos $41 \mathrm{cel} / \mathrm{mm}^{3}$, monomorfos $80 \%$ polimorfos $20 \%$, Proteína $25 \mathrm{mg} / \mathrm{dl}$, glucosa $31 \mathrm{mg} / \mathrm{dl}$, test de ADA 1.9U/L, gram de LCR: negativo. Por los resultados del último LCR alterado se decide continuar tratamiento antibiótico hasta completar 15 días, nuevos exámenes para descartar inmunodepresión. Hemograma: leucocitos $12,600 \mathrm{cel} / \mathrm{mm}^{3}$, segmentados $80 \%$, abastonados $9 \%$, complemento C3 $130 \mathrm{mg} / \mathrm{dl}$ (VN 90-130 mg/dl), Inmunoglobulina IgA $307 \mathrm{mg} / \mathrm{dl}$ (VN 10-40), 
Inmunoglobulina IgG 1209 mg/dl (VN 700-1600), Inmunoglobulina IgM $179 \mathrm{mg} / \mathrm{dl}$ (40-120), Inmunoglobulina lgE $402 \mathrm{mg} / \mathrm{dl}$ (menos de 52). Citometria de Flujo, Linfocitos T CD4: $878 \mathrm{cel} / \mathrm{mm}^{3}$ (300 - 2000 cel. $/ \mathrm{mm}^{3}$ ), Linfocitos T CD8: 731 cel./mm (300-1800), relación T CD4/CD8 1.2 (VN 0.5 - 1.6), linfocitos B CD19+: 201cel/mm $\mathrm{mm}^{3}$ (VN 200 a 1600). El 13 de julio el laboratorio de microbiología reporta que el cultivo de LCR fue negativo.

\section{DISCUSIÓN}

El paciente presentó síntomas de meningitis aguda, realizándose procedimientos como la TAC de cerebro y estudio del LCR previó al inicio de antibióticos; sin embargo, el uso de antibióticos pudo haberse iniciado antes de la PL o la TAC si la progresión rápida de la enfermedad era evidente(6). Así, tener beneficio en el tratamiento inmediato, considerando que otros estudios demuestran eventos desfavorables ante un retraso de tratamiento.

Con respecto a la combinación de antibiótico usado como fue la ceftriaxona y vancomicina, es discutible el uso de vancomicina; según, guías internacionales estaría indicado si existiera prevalencia alta local de resistencia del neumococo a la ceftriaxona (7), en el caso presentado el neumococo aislado era susceptible a ceftriaxona , por lo que debió excluirse en el esquema antibiótico a la vancomicina, al menos al conocerse los resultados de cultivo del LCR. Estaría pendiente realizar estudios epidemiológicos de resistencia bacteriana a antibióticos del neumococo para confirmar la indicación de terapia doble de manera rutinaria. Por otro lado muy acertado el uso de corticoterapia recomendada por las guías de Infectious Diseases Society of America, the European Federation of Neurological Sciences, y the British Infection Society (8-10).

El daño pulmonar y abdominal por neumococo es ocasionado por la bacteriemia a partir de vías respiratorias; al parecer, es frecuente si consideramos que la peritonitis primaria más frecuente en América latina es ocasionada por neumococo, siendo más frecuente en niñas (11). Sin embargo los múltiples ganglios en el mesenterio y región cervical bilateral ameritan continuar estudios según evolución, pudiendo corresponder a procesos de infecciones respiratorias bacterianas o virales como Yersinia enteroclítica, Yersinia pseudotuberculosis, Salmonella spp, Shigella spp, Bartonella spp, Mycobacterium spp., adenovirus, Campilobacter yeyuni, Epstein barr, entre otros siendo la mayoría autolimitadas.

Los pacientes en riesgo para ENI son los menores de 2 años, los adultos mayores y los inmunocomprometidos (12). De allí la importancia de descartar comorbilidades o coinfecciones como la tuberculosis o inmunodepresión, en nuestro paciente los exámenes no determinó comorbilidad, ni inmunodresión celular o humoral, estando pendiente cultivo para tuberculosis.

El Perú adopta medidas de prevención contra el neumococo con la vacuna según lineamientos de la OMS (13), la inclusión de la vacuna antineumocócica en el calendario Nacional de Inmunizaciones se realizó desde el año 2008 con la VCP 7-valente, a partir del 2012 se vacunó con la VCP 10-valente, para este año (2016) el Ministerio de Salud dispone de la VCP13-valente según el esquema de dos dosis primarias más una dosis de refuerzo $(2+1)$ aplicadas a los 2, 4 y 12 meses de edad $(14,15)$.

Debemos mencionar que la paciente nunca recibió vacuna contra el neumococo al no estar disponible cuando tenía edad para inmunizarse. La cepa o serotipo 11 A aislada en el paciente no está incorporada en la vacuna VCP 10-valente (empleada por MINSA en momentos de ocurrencia del caso), por que incluye solo las cepas 4, 5, 6b, 7f, 9v, 14, 18c, 19f, $23 \mathrm{f}$ responsables de la mayoría de las enfermedades invasivas conocidas según estudios en la era pre vacunal.

Al parecer estamos frente a un fenómeno de adaptación del serotipo, esto conlleva a una reducción de enfermedad neumococócica por serotipos vacunales tanto en niños como adultos mayores y un incremento de serotipos no vacunales según estudios con VCP 7 -valente $(2,16)$.

Intentando realizar actividad de vigilancia se solicitó al servicio de microbiología del INSN-Breña un informe de los serotipos circulantes en los últimos 2 meses que estén ocasionando enfermedad severa en niños hospitalizados en el INSN-Breña, obteniendo lo siguiente: paciente con iniciales IPP y MGH ambos 
con diagnóstico de ingreso de neumonía con aislamiento en hemocultivo el serotipo $19 \mathrm{~A}$. información adicional que nos permite descartar la sospecha de un brote de la cepa aislada $11 \mathrm{~A}$.

Por lo mencionado, sugerimos realizar estudios de vigilancia epidemiológica en el INSN ante la necesidad de conocer los cambios clínico epidemiológico y la distribución de serotipos de neumococo después de la introducción de la vacuna, por ser hospital de referencia nacional.

En concusión se presenta un caso de ENI en una niña de 10 años no inmunosuprimida, no expuesta a vacuna contra neumococo previamente, con una cepa la $11 \mathrm{~A}$ no considerada como frecuente para ENI con resistencia a penicilina, con una evolución favorable al tratamiento antibiótico.

\section{REFERENCIAS BIBLIOGRÁFICAS}

1. Behrman E., Kliegmen R., Jonson H. Nelson Textbook of Pediatrics, 17th ed, Elsevier -Saunder 2004.

2. Drijkoningen JJ, Rohde GG. Pneumococcal infection in adults: burden of disease. Clinical microbiology and infection : the official publication of the European Society of Clinical Microbiology and Infectious Diseases. 2014;20 Suppl 5:45-51.

3. Centers for Disease Control and Prevention. Pneumococcal Disease.Epidemiology and Prevention of Vaccine-Preventable Diseases [Internet]. 12th ed. Washington DC: Public Health Foundation,; 2012 [cited 2012 Aug 1]. page 233-48. Available from:http://www.cdc.gov/vaccines/ pubs/pinkbook/ downloads/ pneumo.pdf.

4. World Health Organization. Weekly epidemiological record 2012; 14, 87: 129-144. .

5. Ministerio de Salud, Dirección General de Epidemiología. Análisis de la Situación de Salud del Perú. 2010.

6. Diederik van de Beek. Et al. Community-Acquired Bacterial Meningitis in Adults. N Engl J Med 2006;354:44-53.

7. Saez-Llorens, X., and G. H. McCracken, Jr. 2003. Bacterial meningitis in children. Lancet 361:2139-2148.

8. Chaudhuri, A., P. Martinez-Martin, P. G. Kennedy, S. R. Andrew, P. Portegies, M. Bojar, and I. Steiner. 2008. EFNS guideline on the management of community-acquired bacterial meningitis: report of an EFNS task force on acute bacterial meningitis in older children and adults. Eur. J. Neurol.15:649-659.

9. Heyderman, R. S., H. P. Lambert, I. O'Sullivan, J. M. Stuart, B. L. Taylor, and R. A. Wall. 2003. Early management of suspected bacterial meningitis and meningococcal septicaemia in adults. J. Infect. 46:75-77.

10. Tunkel, A. R., B. J. Hartman, S. L. Kaplan, B. A. Kaufman, K. L. Roos, W. M. Scheld, and R. J. Whitley. 2004. Practice guidelines for the management of bacterial meningitis. Clin. Infect. Dis. 39:1267-1284.

11. Andrade AL, Toscano CM, Minamisava R, Costa PS, Andrade JG. Pneumococcal disease manifestation in children before and after vaccination: what's new? Vaccine. 2011;29 Suppl 3:C214.

12. Gladstone RA, Jefferies JM, Faust SN, Clarke SC. Continued control of pneumococcal disease in the UK - the impact of vaccination. Journal of medical microbiology. 2011;60(Pt 1):1-8.

13. World Health Organization. Pneumococcal conjugate vaccine for childhood immunization--WHO position paper.Wkly. Epidemiol. Rec. 2007 Mar 23;82(12):93-104.

14. Organización Panamericana de la Salud. Informe Regional de SIREVA II, 2011: datos por país y por grupos de edad sobre las características de los aislamientos de Streptococcus pneumoniae, Haemophilus influenzae y Neisseria meningitidis en procesos invasores. 2012. .

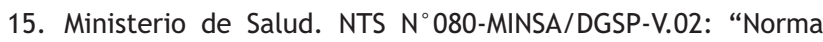
Técnica de Salud que establece el Esquema Nacional de Vacunación" [Internet]. 2011 [cited 2013 Jan 4]. Available from: ftp://ftp2.minsa.gob.pe/normaslegales/2011/RM0702011-MINSA1.PD

16. Miller E, Andrews NJ, Waight PA, Slack MP, George RC. Herd immunity and serotype replacement 4 years after sevenvalent pneumococcal conjugate vaccination in England and Wales: an observational cohort study. The Lancet Infectious diseases. 2011;11(10):760-8.

Fuente de Financiamiento

Este artículo ha sido financiado por los autores.

Conflictos de interés

Los autores declaran no tener conflicto de interés en la presentación de este artículo.

Correspondencia:

Edwin Albino H. Miranda Choque

Dirección: Pachacutec 910, Jesús María

Teléfono: 968544431

Correo electrónico: emirandach@hotmail.com

Recibido: 03 de Diciembre de 2015 Aprobado: 24 de Enero de 2016 